\title{
On Effective Non-vanishing of Weil Divisors on Algebraic Surfaces *
}

\author{
Qihong Xie
}

\begin{abstract}
We give a counterexample and some conclusions for effective nonvanishing of Weil divisors on algebraic surfaces.
\end{abstract}

The basepoint-free theorem plays a crucial role in the Minimal Model Program since it yields a basepoint-free complete linear system, hence an extremal contraction morphism. More precisely, it says that if $D$ is a nef Cartier divisor such that the difference between $D$ and the $\log$ canonical divisor is nef and big, then the linear system $|m D|$ is basepoint-free for sufficiently large integer $\mathrm{m}$ (cf. [6, 7]). Obviously, it is natural and interesting to investigate the non-emptyness of the linear system $|D|$. Therefore, the following so-called Effective Non-vanishing Conjecture has been put forward formally by Yujiro Kawamata (cf. [5] ).

Conjecture 1. Let $X$ be a complete normal variety, $B$ an effective $\mathbb{R}$-divisor on $X$ such that the pair $(X, B)$ is Kawamata log terminal, and $D$ a Cartier divisor on $X$. Assume that $D$ is nef and that $D-\left(K_{X}+B\right)$ is nef and big. Then $H^{0}(X, D) \neq 0$.

By the Kawamata-Viehweg vanishing theorem (cf. [6]), we have $H^{p}(X, D)$ $=0$ for any positive integer $p$. Thus the condition $H^{0}(X, D) \neq 0$ is equivalent to saying that $\chi(X, D) \neq 0$. Since the Riemann-Roch formula of the line bundle $\mathcal{O}_{X}(D)$ depends only on the intersections of Chern classes of $X$ and $D$, we can say that the Effective Non-vanishing Conjecture is indeed a topological problem in a sense of complex geometry. This conjecture is of great importance by at least two reasons. At first, it provides a good tool to study the geometry of Fano varieties (cf. [1]). Secondly, it is closely related to the general elephant problem (cf. [10]).

Kawamata has proven that the Effective Non-vanishing Conjecture holds for all log surfaces with only Kawamata log terminal singularities (cf. [5]). In dimension three, this conjecture is more complicated, only a few results are known.

It is also interesting to consider a new problem by replacing Cartier with Q-Cartier.

*2000 Mathematics Subject Classification Primary 14J25; Secondary 14J45, 14E30. 
Problem 2. Notation are the same as in Conjecture 1. Assume that $D$ is a nef $\mathbb{Q}$-Cartier Weil divisor on $X$, such that $D-\left(K_{X}+B\right)$ is nef and big. Does $H^{0}(X, D) \neq 0$ hold?

Unfortunately, this problem does not hold generally. In dimension three, Fletcher has given the following famous example (cf. 44).

Example 3. A general weighted complete intersection $X_{12,14}$ in $\mathbb{P}(2,3,4,5,6,7)$ is a terminal $\mathbb{Q}$-Fano threefold of Picard number one with $H^{0}\left(-K_{X}\right)=0$. This variety has the following isolated singularities: one of type $\frac{1}{5}(4,1,2)$, two of type $\frac{1}{3}(2,1,1)$, and seven of type $\frac{1}{2}(1,1,1)$.

In dimension two, such a counterexample of Problem 2 also exists, which is Example 7 given later in this paper. At first, we give some notation and definitions for the convenience of the reader.

Definition 4. A normal surface $X$ is said to be log terminal (resp. canonical), if the following conditions are satisfied.

(1) $K_{X}$ is a $\mathbb{Q}$-Cartier divisor, i.e., there exists some positive integer $r$ such that $r K_{X}$ is Cartier, and

(2) For any resolution $f: Y \rightarrow X$, if we write $K_{Y}=f^{*} K_{X}+\sum \alpha_{i} E_{i}$, where $E_{i}$ are irreducible exceptional curves of $f$, then $\alpha_{i}>-1$ (resp. $\alpha_{i} \geq 0$ ) for all $i$.

The index of $X$ is, by definition, the smallest positive integer $r$ such that $r K_{X}$ is Cartier. If the index of $X$ is one, then $X$ is said to be Gorenstein. If the resolution $f$ is minimal, i.e., $K_{Y}$ is $f$-nef, then we have that $\alpha_{i} \leq 0$ for all $i$ since the intersection matrix $\left(E_{i} . E_{j}\right)$ is negative definite.

Definition 5. A normal surface $X$ is called a log del Pezzo surface, if $X$ has only $\log$ terminal singularities and $-K_{X}$ is ample.

For a $\mathbb{Q}$-divisor $D=\sum a_{i} D_{i}$, the round up (resp. round down) of $D$ is defined to be $\lceil D\rceil=\sum\left\lceil a_{i}\right\rceil D_{i}$ (resp. $\left.\lfloor D\rfloor=\sum\left\lfloor a_{i}\right\rfloor D_{i}\right)$, where $\lceil a\rceil$ (resp. $\lfloor a\rfloor$ ) is the smallest integer not less (resp. the largest integer not greater) than $a$.

Proposition 6. Let $X$ be a log terminal surface, $f: Y \rightarrow X$ the minimal resolution of $X$. Assume that $K_{Y}=f^{*} K_{X}+\sum \alpha_{i} E_{i}$, where $-1<\alpha_{i} \leq 0$ for all $i$. Then for each integer $n \geq 0, \chi\left(-n K_{X}\right):=\chi\left(X, \mathcal{O}_{X}\left(-n K_{X}\right)\right)$ is equal to

$\frac{n(n+1)}{2} K_{Y}^{2}-\frac{2 n+1}{2} K_{Y} \cdot\left(\sum\left\lceil(n+1) \alpha_{i}\right\rceil E_{i}\right)+\frac{1}{2}\left(\sum\left\lceil(n+1) \alpha_{i}\right\rceil E_{i}\right)^{2}+\chi\left(\mathcal{O}_{Y}\right)$.

Proof. Let $D=-n K_{X}$. Then we have

$$
\begin{aligned}
f^{*}\left(D-K_{X}\right) & =-(n+1) f^{*} K_{X}=-(n+1)\left(K_{Y}-\sum \alpha_{i} E_{i}\right) \\
& =\left(-n K_{Y}+(n+1) \sum \alpha_{i} E_{i}\right)-K_{Y} .
\end{aligned}
$$


We define $\widetilde{D}, \widetilde{B}$ as follows:

$$
\begin{aligned}
\widetilde{D} & =\left\lceil-n K_{Y}+(n+1) \sum \alpha_{i} E_{i}\right\rceil=-n K_{Y}+\sum\left\lceil(n+1) \alpha_{i}\right\rceil E_{i}, \\
\widetilde{B} & =\sum\left(\left\lceil(n+1) \alpha_{i}\right\rceil-(n+1) \alpha_{i}\right) E_{i} .
\end{aligned}
$$

It is easy to see that $f^{*}\left(D-K_{X}\right)=\widetilde{D}-\left(K_{Y}+\widetilde{B}\right)$ is an $f$-nef and $f$-big divisor, and $\widetilde{B}$ has simple normal crossing support. By the KawamataViehweg vanishing theorem, we have $R^{j} f_{*} \mathcal{O}_{Y}(\lceil\widetilde{D}-\widetilde{B}\rceil)=R^{j} f_{*} \mathcal{O}_{Y}(\widetilde{D})=0$ for any positive integer $j$.

Since we have

$$
\begin{aligned}
f^{*} D= & -n K_{Y}+\sum n \alpha_{i} E_{i}=\widetilde{D}+\sum\left(n \alpha_{i}-\left\lceil(n+1) \alpha_{i}\right\rceil\right) E_{i}, \\
& n \alpha_{i}-\left\lceil(n+1) \alpha_{i}\right\rceil<(n+1) \alpha_{i}-\left\lceil(n+1) \alpha_{i}\right\rceil+1 \leq 1,
\end{aligned}
$$

there exists a $\mathbb{Q}$-divisor $H=\sum h_{i} E_{i}$ on $Y$ such that $0 \leq h_{i}<1$ and $h_{i}-\left(n \alpha_{i}-\left\lceil(n+1) \alpha_{i}\right\rceil\right) \geq 0$ for each $i$. For a $\mathbb{Q}$-divisor $M$ on $Y$, the sheaf $\mathcal{O}_{Y}(M)$ is defined to be $\mathcal{O}_{Y}(\lfloor M\rfloor)$. Thus $f_{*} \mathcal{O}_{Y}(\widetilde{D})=f_{*} \mathcal{O}_{Y}(\widetilde{D}+H)=$ $\mathcal{O}_{X}(D)$ by the projection formula. It follows from [3] that we have the Leray spectral sequence

$$
E_{2}^{i, j}=H^{i}\left(X, R^{j} f_{*} \mathcal{O}_{Y}(\widetilde{D})\right) \Rightarrow H^{i+j}\left(Y, \mathcal{O}_{Y}(\widetilde{D})\right)
$$

Since $E_{2}^{i, j}=0$ for all $i \geq 0$ and $j>0, E_{2}^{i, 0} \cong E_{\infty}^{i, 0}$ implies that $h^{i}\left(X, \mathcal{O}_{X}(D)\right)=$ $h^{i}\left(Y, \mathcal{O}_{Y}(\widetilde{D})\right)$ for all $i \geq 0$, hence $\chi\left(X, \mathcal{O}_{X}(D)\right)=\chi\left(Y, \mathcal{O}_{Y}(\widetilde{D})\right)$. Therefore we have

$$
\begin{aligned}
& \chi\left(-n K_{X}\right) \\
= & \chi\left(X, \mathcal{O}_{X}(D)\right)=\chi\left(Y, \mathcal{O}_{Y}(\widetilde{D})\right)=\frac{1}{2} \widetilde{D}\left(\widetilde{D}-K_{Y}\right)+\chi\left(\mathcal{O}_{Y}\right) \\
= & \frac{1}{2}\left(-n K_{Y}+\sum\left\lceil(n+1) \alpha_{i}\right\rceil E_{i}\right)\left(-(n+1) K_{Y}+\sum\left\lceil(n+1) \alpha_{i}\right\rceil E_{i}\right)+\chi\left(\mathcal{O}_{Y}\right) \\
= & \frac{n(n+1)}{2} K_{Y}^{2}-\frac{2 n+1}{2} K_{Y} \cdot\left(\sum\left\lceil(n+1) \alpha_{i}\right\rceil E_{i}\right)+\frac{1}{2}\left(\sum\left\lceil(n+1) \alpha_{i}\right\rceil E_{i}\right)^{2}+\chi\left(\mathcal{O}_{Y}\right) .
\end{aligned}
$$

Example 7. There exists a log del Pezzo surface $S$ of Picard number one such that $H^{0}\left(S,-K_{S}\right)=0$.

Let $B$ be a smooth conic in $\mathbb{P}^{2}, D$ a tangent line to $B$ at $d$, and $A$ a secant line intersecting $B$ at $\{a, b\}$. Let $g_{1}: S_{1} \rightarrow \mathbb{P}^{2}$ be the blow-up of $\mathbb{P}^{2}$ along $d \in D$ with an exceptional curve $L_{1}$. By abuse of notation, we always denote the strict transform of a curve by the same symbol. Let $g_{i}: S_{i+1} \rightarrow S_{i}$ be the blow-up of $S_{i}$ along $d \in D$ with exceptional curves $L_{i+1}$ for $i=1,2$. It is easy to see that $L_{1}^{2}=L_{2}^{2}=-2, L_{3}^{2}=-1$. In a similar way, let $g_{4}: S_{4} \rightarrow S_{3}$ be the blow-up of $S_{3} 5$ times along $b \in B$. 
Assume that the exceptional curves of $g_{4}$ are denoted by $M_{1}, M_{2}, M_{3}, M_{4}, M_{5}$ respectively with $M_{1}^{2}=M_{2}^{2}=M_{3}^{2}=M_{4}^{2}=-2$, and $M_{5}^{2}=-1$. Let $g_{5}: \widetilde{S} \rightarrow S_{4}$ be the blow-up of $S_{4} 5$ times along $a \in A$. Assume that the exceptional curves of $g_{5}$ are denoted by $N_{1}, N_{2}, N_{3}, N_{4}, N_{5}$ respectively with $N_{1}^{2}=N_{2}^{2}=N_{3}^{2}=N_{4}^{2}=-2$, and $N_{5}^{2}=-1$. Denote by $g: \widetilde{S} \rightarrow \mathbb{P}^{2}$ the composition of all $g_{i}$.

We consider two reduced connected curves $X_{1}:=D \cup A \cup M_{1} \cup M_{2} \cup$ $M_{3} \cup M_{4}$ and $X_{2}:=L_{1} \cup L_{2} \cup B \cup N_{1} \cup N_{2} \cup N_{3} \cup N_{4}$ on $\widetilde{S}$. It is easy to verify that the intersection matrix associated with $X_{i}$ is negative definite, and the arithmetic genus of any positive cycle with support on $X_{i}$ is nonpositive for $i=1,2$. Thus $X_{1} \cup X_{2}$ is contractible, and there is a contraction $f: \widetilde{S} \rightarrow S$ such that $S$ is a normal projective surface of Picard number one with only two singular points $x_{1}, x_{2}$, which is the contraction point of $X_{1}, X_{2}$ respectively (cf. [2]).

It is easy to see that $\widetilde{S}$ is just the minimal resolution of $S$, and the dual graph $\Gamma$ consists of two connected components corresponding to $x_{1}, x_{2}$ respectively.

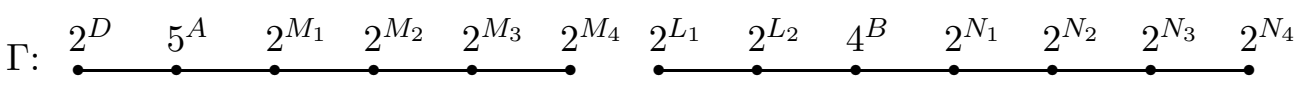

where $2^{D}$ denotes the $(-2)$-curve on $\widetilde{S}$ which is the strict transform of $D$, and so on.

By an elementary calculation, we have

$$
\begin{aligned}
K_{\widetilde{S}} & =f^{*} K_{S}-\frac{15}{37} D-\frac{30}{37} A-\frac{24}{37} M_{1}-\frac{18}{37} M_{2}-\frac{12}{37} M_{3}-\frac{6}{37} M_{4} \\
& -\frac{5}{19} L_{1}-\frac{10}{19} L_{2}-\frac{15}{19} B-\frac{12}{19} N_{1}-\frac{9}{19} N_{2}-\frac{6}{19} N_{3}-\frac{3}{19} N_{4}
\end{aligned}
$$

It follows easily that $K_{S}^{2}=8 /(37 \times 19)>0$, then $-K_{S}$ is ample since $S$ is rational and $\rho(S)=1$. Furthermore, we have $H^{0}\left(S,-n K_{S}\right)=0$ for $1 \leq n \leq 5$, but $H^{0}\left(S,-6 K_{S}\right) \cong \mathbb{C}$ by Proposition 6

Remark 8. For a log del Pezzo surface $X$, we define $\tau(X)$ to be the smallest positive integer $\tau$ such that $H^{0}\left(X,-\tau K_{X}\right) \neq 0$. It is easy to see that the index $r(X)$ of $X$ is not less than $\tau(X)$. The study of complements on surfaces is helpful to solve the non-vanishing of $H^{0}\left(X,-n K_{X}\right)$ for log del Pezzo surfaces. Shokurov proved that there exists a positive integer $N$ such that $\tau(X) \leq N$ for all log del Pezzo surfaces (cf. 11]). In particular, Prokhorov used the same method to yield that $H^{0}\left(X,-n K_{X}\right) \neq 0$ for some $n \in \mathcal{R}_{2}:=\{1,2,3,4,6\}$, if $K_{X}^{2}>4$ (cf. 9]).

Example[7illustrates that Problem[2] does not hold generally for surfaces. But, when some reasonable condition is assumed, we hope that the effective non-vanishing of Weil divisors holds. We give the following theorem as a special example. 
Theorem 9. Let $X$ be a Gorenstein log del Pezzo surface of Picard number one, $D$ a nef and big $\mathbb{Q}$-Cartier Weil divisor on $X$. Then $H^{0}(X, D) \neq 0$.

It is easy to see that $X$ has only canonical singularities. The condition $\rho(X)=1$ enables us to list all types of its singularities.

Lemma 10. Let $X$ be a Gorenstein log del Pezzo surface with $\rho(X)=1$. Then its singularity type is one of the following:

$A_{1}, A_{1}+A_{2}, A_{4}, 2 A_{1}+A_{3}, D_{5}, A_{1}+A_{5}, 3 A_{2}, E_{6}, 3 A_{1}+D_{4}, A_{7}, A_{1}+D_{6}$, $E_{7}, A_{1}+2 A_{3}, A_{2}+A_{5}, D_{8}, 2 A_{1}+D_{6}, E_{8}, A_{1}+E_{7}, A_{1}+A_{7}, 2 A_{4}, A_{8}$, $A_{1}+A_{2}+A_{5}, A_{2}+E_{6}, A_{3}+D_{5}, 4 A_{2}, 2 A_{1}+2 A_{3}, 2 D_{4}$.

Proof. $X$ is said to be of type, for example, $2 A_{1}+A_{3}$, if $X$ has three singular points, two of type $A_{1}$, and one of type $A_{3}$. We refer to [8] for the proof.

Definition 11. The quotient $X=\mathbb{A}^{n} / \mu_{r}$ is said to be of cyclic quotient singularity of type $\frac{1}{r}\left(a_{1}, \cdots, a_{n}\right)$, if $\mu_{r}$ acts on $\mathbb{A}^{n}$ by

$$
\mu_{r} \ni \varepsilon:\left(x_{1}, \cdots, x_{n}\right) \mapsto\left(\varepsilon^{a_{1}} x_{1}, \cdots, \varepsilon^{a_{n}} x_{n}\right) .
$$

Let $\pi: \mathbb{A}^{n} \rightarrow X$ be the quotient morphism. Then the group $\mu_{r}$ acts on $\pi_{*} \mathcal{O}_{\mathbb{A}^{n}}$, and so decomposes it into $r$ eigensheaves $\mathcal{L}_{i}=\left\{f \mid \varepsilon(f)=\varepsilon^{i} \cdot f\right.$ for all $\left.\varepsilon \in \mu_{r}\right\}$ for $i=0, \cdots, r-1$. A singularity $p \in X$ with a Weil divisor $D$ is said to be of cyclic quotient singularity of type $i\left(\frac{1}{r}\left(a_{1}, \cdots, a_{n}\right)\right)$, if locally, $p \in X$ is isomorphic to a point of type $\frac{1}{r}\left(a_{1}, \cdots, a_{n}\right)$, and $\mathcal{O}_{X}(D) \cong \mathcal{L}_{i}$.

There are two keypoints in the proof of Theorem 9. First, one canonical singularity can be considered as the cyclic quotient of another canonical singularity by using the local cyclic cover. Second, we can calculate the Euler characteristic of a Weil divisor, thanks to the Singular Riemann-Roch Formula.

The following lemma is helpful, which can be found in [10].

Lemma 12. Let $Q \in Y:(f=0) \subset \mathbb{A}^{3}$ be a surface germ. Assume that there is an action of $\mu_{r}$ on $Y$ free outside $Q$. Let $p \in X=(Q \in Y) / \mu_{r}$ be the surface germ and $\pi: Y \rightarrow X$ the corresponding cyclic cover. If $p \in X$ is canonical, then $Q \in Y$ is also canonical and $\pi: Y \rightarrow X$ is exactly one of the following types.

\begin{tabular}{|c|c|l|l|l|}
\hline & $r$ & Type & $f$ & Description \\
\hline$(1)$ & any & $\frac{1}{r}(1,-1,0)$ & $x y+z^{n}$ & $A_{n-1} \stackrel{r: 1}{\rightarrow} A_{r n-1}$ \\
$(2)$ & 4 & $\frac{1}{4}(1,3,2)$ & $x^{2}+y^{2}+z^{2 n-1}$ & $A_{2 n-2} \stackrel{4: 1}{\rightarrow} D_{2 n+1}$ \\
$(3)$ & 2 & $\frac{1}{2}(0,1,1)$ & $x^{2}+y^{2}+z^{2 n}$ & $A_{2 n-1} \stackrel{2: 1}{\rightarrow} D_{n+2}$ \\
$(4)$ & 3 & $\frac{1}{3}(0,1,2)$ & $x^{2}+y^{3}+z^{3}$ & $D_{4} \stackrel{3: 1}{\rightarrow} E_{6}$ \\
$(5)$ & 2 & $\frac{1}{2}(1,1,0)$ & $x^{2}+y^{2} z+z^{n}$ & $D_{n+1} \stackrel{2: 1}{\rightarrow} D_{2 n}$ \\
$(6)$ & 2 & $\frac{1}{2}(1,0,1)$ & $x^{2}+y^{3}+z^{4}$ & $E_{6} \stackrel{2: 1}{\rightarrow} E_{7}$ \\
\hline
\end{tabular}


Proof of Theorem 9. If $D$ is a Cartier divisor, then the non-vanishing follows from [5]. Now assume that $D$ is not Cartier. For the reflexive sheaf $\mathcal{O}_{X}(D)$, we have the following Singular Riemann-Roch Formula (cf. 10]):

$$
\chi\left(X, \mathcal{O}_{X}(D)\right)=\frac{1}{2} D \cdot\left(D-K_{X}\right)+\chi\left(\mathcal{O}_{X}\right)+\sum_{p \in X} c_{p}(D),
$$

where $c_{p}(D) \in \mathbb{Q}$ is a contribution due to the singularity of $\mathcal{O}_{X}(D)$ at $p$, the sum is taken over the points $p \in X$ at which $D$ is not Cartier.

If $p \in X$ and $D$ is of type $i\left(\frac{1}{r}(1,-1)\right)$, then $c_{p}(D)=-i(r-i) / 2 r$. For other cases, we use the suitable $Q$-smoothing of $X$, then there is a flat deformation $\left\{X_{\lambda}, D_{\lambda}\right\}$ of $\{X, D\}$ such that $X_{\lambda}$ has only cyclic quotient singularities. Thus $c_{p}(D)$ is equal to the sum of the contributions of cyclic quotient singularities of $X_{\lambda}$. The concrete conclusions are listed below.

\begin{tabular}{|c|c|c|}
\hline & $X_{\lambda}$ & $Q$-smoothing points \\
\hline$(1)$ & $f+\lambda z$ & $\mathrm{n}$ \\
$(2)$ & $f+\lambda z$ & $2 \mathrm{n}+1$ \\
$(3)$ & $f+\lambda x$ & 2 \\
$(4)$ & $f+\lambda x$ & 2 \\
$(5)$ & $f+\lambda z$ & $\mathrm{n}$ \\
$(6)$ & $f+\lambda y$ & 3 \\
\hline
\end{tabular}

Since $-K_{X}$ is an ample Cartier divisor and $D$ is a nef and big Weil divisor, it is easy to show that $\chi\left(\mathcal{O}_{X}\right)=1$ and $D .\left(-K_{X}\right) \geq 1$. Thus $h^{0}(X, D) \geq D^{2} / 2+3 / 2+\sum_{p \in X} c_{p}(D)$.

Next, we will estimate $c_{p}(D)$ for a given germ $p \in X$ at which $D$ is not Cartier. Since the cyclic covers in Lemma 12 can be constructed locally, and the contribution $c_{p}(D)$ depends only on the local analytic type of $p \in X$ and $D$, we can take $\pi: Y \rightarrow X$ to be a suitable cyclic cover in Lemma 12 and estimate $c_{p}(D)$ as follows:

Let $p \in X$ be of type $A_{n-1}$. Then $p \in X$ is a cyclic quotient singularity of type $\frac{1}{n}(1,-1)$. By definition, we have $c_{p}(D)=-i_{0}\left(n-i_{0}\right) / 2 n$ for some integer $i_{0} \in[0, n]$. If $n=2 k$, then $c_{p}(D) \geq-k / 4$. If $n=2 k+1$, then $c_{p}(D) \geq-k(k+1) / 2(2 k+1)$.

Let $p \in X$ be of type $D_{n+2}$. We may consider $p \in X$ as a cyclic quotient of type (3). Then $c_{p}(D) \geq-1 / 4 \times 2=-1 / 2$.

Let $p \in X$ be of type $E_{6}$. We may consider $p \in X$ as a cyclic quotient of type (4). Then $c_{p}(D) \geq-1 / 3 \times 2=-2 / 3$.

Let $p \in X$ be of type $E_{7}$. We may consider $p \in X$ as a cyclic quotient of type (6). Then $c_{p}(D) \geq-1 / 4 \times 3=-3 / 4$.

Since $E_{8}$ does not appear in the table of Lemma $12 p \in X$ could not be of type $E_{8}$ provided that $D$ is not Cartier at $p$.

It is easy to check for all cases in Lemma 10 that $\sum_{p \in X} c_{p}(D) \geq-3 / 2$. Hence $h^{0}(X, D) \geq D^{2} / 2>0$. 
Acknowledgment. I would like to express my gratitude to Professor Yujiro Kawamata for his valuable advice and warm encouragement. I also thank Professor S. A. Kudryavtsev for informing me some known results in Remark 8, and Dr. Hokuto Uehara for stimulating discussions on this paper.

\section{References}

[1] F. Ambro, Ladders on Fano varieties, Algebraic geometry, 9. J. Math. Sci. (New York), 94(1999), no. 1, 1126-1135.

[2] M. Artin, Some numerical criteria for contractability of curves on algebraic surfaces, Amer. J. Math., 84(1962), 485-496.

[3] G. Bredon, Sheaf theory, Springer, 1997.

[4] A. R. Fletcher, Working with weighted complete intersections, Explicit birational geometry of 3-folds, Lond. Math. Soc. Lect. Note Ser., 281(2000), 101-173.

[5] Y. Kawamata, On effective non-vanishing and base-point-freeness, Asian J. Math., 4(2000), 173-182.

[6] Y. Kawamata, K. Matsuda, K. Matsuki, Introduction to the minimal model problem, Alg. Geom. Sendai 1985, Adv. Stud. in Pure Math., 10(1987), Kinokuniya, Tokyo, 283-360.

[7] J. Kollár, S. Mori, Birational geometry of algebraic varieties, Cambridge Tracts in Math., 134(1998).

[8] M. Miyanishi, D. Q. Zhang, Gorenstein log del Pezzo surfaces of rank one, J. Alg., 118(1988), 63-84.

[9] Y. G. Prokhorov, Lectures on complements on log surfaces, Math. Soc. of Japan Memoirs, 10(2001).

[10] M. Reid, Young person's guide to canonical singularities, Proc. Symp. Pure Math., 46(1987), 345-416.

[11] V. V. Shokurov, Complements on surfaces, Algebraic geometry, 10. J. Math. Sci. (New York), 102(2000), no. 2, 3876-3932.

Graduate School of Mathematical Sciences, University of

Tokyo, 3-8-1 Komaba, Meguro, Tokyo 153-8914, Japan

E-mail address: xqh@ms.u-tokyo.ac.jp 